\title{
¿CÓMO RESPONDER DESDE LA ACADEMIA A LOS RIESGOS QUE PREOCUPAN A LAS ORGANIZACIONES?
}

Guzmán Useche, Hugo Armando; Maldonado Pedroza, Cristian David

¿CÓMO RESPONDER DESDE LA ACADEMIA A LOS RIESGOS QUE PREOCUPAN A LAS

ORGANIZACIONES?

PANORAMA, vol. 15, núm. 28, 2021

Politécnico Grancolombiano, Colombia

Disponible en: https://www.redalyc.org/articulo.oa?id=343965146007

Institución Universitaria Politécnico Grancolombiano

Institución Universitaria Politécnico Grancolombiano

\section{(c) $(1)(9)$}

Esta obra está bajo una Licencia Creative Commons Atribución-NoComercial-SinDerivar 4.0 Internacional. 
PANORAMA, vol. 15, núm. 28, 2021

Politécnico Grancolombiano, Colombia

Recepción: 08 Septiembre 2020 Aprobación: 21 Octubre 2020

Redalyc: https://www.redalyc.org/ articulo.oa?id $=343965146007$
Artículos de investigación científica y tecnológica

¿CÓMO RESPONDER DESDE LA ACADEMIA A LOS RIESGOS
QUE PREOCUPAN A LAS
ORGANIZACIONES?

HOW CAN THE ACADEMY RESPOND TO THE RISKS THAT CONCERN TO THE ORGANIZATIONS?

COMO RESPONDER DA ACADEMIA AOS RISCOS QUE PREOCUPAM AS ORGANIZAÇÕES?

Hugo Armando Guzmán Useche haguzmanu@poligran.edu.co Institución Universitaria Politécnico Grancolombiano., Colombia

Cristian David Maldonado Pedroza cdmaldonado@poligran.edu.co Institución Universitaria Politécnico Grancolombiano., Colombia

Resumen: Riesgo es un término empleado para referirse a sucesos que pueden afectar el cumplimiento de los objetivos de una empresa. Desde la academia se vienen desarrollando alternativas educativas que permitan abordar el estudio de los riesgos, con el fin de apoyar al mercado asegurador colombiano y brindar a las empresas las herramientas necesarias para su prevención y mitigación.

El presente documento busca identificar, con base en publicaciones de instituciones especializadas en el estudio de la gestión del riesgo en las organizaciones y en la opinión de estudiantes y directivos del sector asegurador en Colombia, cuáles son los riesgos, en el contexto actual de los negocios, que pueden generar algún impacto sobre los objetivos de una organización. Esta identificación se usa como marco de comparación con la oferta académica posgradual colombiana, para poder determinar la brecha que hay entre las necesidades del entorno y la preparación de los profesionales especializados en riesgos. Se pudo concluir que el carácter dinámico de los riesgos exige, desde las comunidades educativas, contar con programas que permitan formar profesionales que sean capaces de administrar las organizaciones en estos nuevos contextos, haciendo énfasis en la naturaleza de integralidad que debe permear la gestión de riesgos.

Palabras clave: Riesgo, estudios de posgrado, riesgos globales, gestión de riesgos, riesgos asegurables, riesgos no asegurables, enseñanza.

Abstract: The term risk is associated to events that can affect the fulfillment of a company's objectives. In our country, postgraduate programs have been developing educational alternatives that allow addressing the study of risk management, to support the Colombian insurance market and providing companies with the necessary tools for the prevention and mitigation of risks.

This document seeks to identify, based on publications of institutions specialized in the study of risk management in organizations and on the opinion of students and executives of the insurance sector in Colombia, what the risks are, in the current business context , which can generate some impact on the objectives of an organization. This identification is used as a framework for comparison with the Colombian postgraduate academic offer, to determine the gap between the needs of the environment and the preparation of professionals specialized in risks. 
It was concluded that the dynamic nature of risks requires educational communities to have programs that train professionals who can manage organizations in these new contexts, emphasizing in an integrated risk management point of view.

Keywords: Risk, postgraduate studies, global risks, risk management, insurable risks, uninsurable risks, teaching.

Resumo: Risco é um termo usado para se referir a eventos que podem afetar a realização dos objetivos de uma empresa. A academia vem desenvolvendo alternativas educacionais que permitem o estudo dos riscos a serem tratados, a fim de apoiar o mercado segurador colombiano e fornecer às empresas as ferramentas necessárias para sua prevenção e mitigação.

Este documento busca identificar, a partir de publicações de instituições especializadas no estudo da gestão de riscos nas organizações e na opinião de estudantes e executivos do setor de seguros na Colômbia, quais são os riscos, no atual contexto empresarial, o que pode gerar algum impacto nos objetivos de uma organização. Esta identificação é utilizada como marco de comparação com a oferta acadêmica de pós-graduação colombiana, a fim de determinar a lacuna entre as necessidades do meio ambiente e a formação de profissionais especializados em riscos.

Foi possível concluir que a natureza dinâmica dos riscos exige, das comunidades educativas, programas que possibilitem a formação de profissionais que sejam capazes de gerir organizações nestes novos contextos, enfatizando o caráter da integralidade que deve permear a gestão dos riscos.

Palavras-chave: Risco, estudos de pós-graduação, riscos globais, gestão de riscos, riscos seguráveis, riscos não seguráveis, Ensino.

\section{INTRODUCCIÓN}

Con el presente documento se pretende reconocer las competencias requeridas en el mercado colombiano, en relación con la educación sobre gestión del riesgo, basado en tendencias mundiales, regionales y locales. El estudio pretende que una persona que quiera aprender sobre el tema lo haga de manera integral, involucrando las áreas de interés en una empresa.

Para abordar el problema bajo estudio se parte de la especialización en Gerencia de Riesgos y seguros del Politécnico Grancolombiano, que nació como un complemento a la Tecnología de Seguros que dicha institución contenía dentro de sus programas desde su fundación. En su momento, los contenidos del programa de especialización correspondían a la realidad existente en materia de riesgos y pólizas de seguros. Sin embargo, en el contexto actual, con los cambios tecnológicos, el internet de las cosas, los riesgos cibernéticos, la inteligencia artificial, la robótica, entre otros aspectos, resulta oportuno preguntarse si los contenidos de la oferta académica son pertinentes o si por el contrario requieren ser actualizados y/o replanteados, para responder a las demandas de un sector empresarial afectado cada vez más por situaciones complejas.

Para reconocer la importancia que la especialización en Gerencia de Riesgos y seguros tiene para el mercado colombiano podemos indicar que, en el año 2020, dicha especialización contaba con 14 módulos, 64\% de ellos dedicados al conocimiento específico sobre seguros y riesgos asegurables, y los 36\% restantes orientados a habilidades de liderazgo, dirección y análisis económicos y del mercado asegurador, que le permitían al estudiante gestionar los riesgos asegurables de cualquier 
organización, o trabajar dentro de una compañía aseguradora en sus áreas de dirección, suscripción y riesgos.

En Bogotá, el Politécnico Grancolombiano es reconocido como una institución que enseña sobre riesgos asegurables y pólizas de seguros; existen en el mercado dos propuestas más que abordan la administración de los riesgos: la maestría en Gestión integral del riesgo, de la Universidad Externado de Colombia (en Bogotá), y la maestría en Administración de riesgos, de EAFIT (en Medellín).

Las maestrías antes mencionadas abordan el concepto de riesgo, metodologías de valoración y tratamiento, su gestión, desde la alta dirección, sus finanzas, la interrupción del negocio y su reputación, como temas comunes, dejando la oportunidad de abordar el riesgo desde otras perspectivas más específicas, como el manejo del recurso humano, el cambio climático, la incursión de nuevas tecnologías, quiebra de clientes, problemas medio ambientales, la relación del ser humano y su entorno, entre otros riesgos que puedan también tener relación con las actividades de la empresa y sus objetivos estratégicos.

Para aprender solo sobre pólizas de seguros existen también propuestas distintas a la del Politécnico Grancolombiano; instituciones como la Universidad de Nuestra Señora del Rosario, Externado, Pontificia Universidad Javeriana, EAFIT, Universidad de La Sabana, representan esas otras alternativas.

La pregunta es: ¿Cuáles son esos riesgos relevantes que deberían tenerse en cuenta para un programa de posgrado, teniendo como base el contexto empresarial contemporáneo?

Para responder a este interrogante, el presente artículo se estructura en tres partes, además de esta introducción: en la primera, se construye un marco referencial acerca del estudio del riesgo desde la perspectiva de las organizaciones, buscando detallar un inventario de riesgos que, de acuerdo con diferentes centros de estudios especializados, concentran la preocupación de las empresas a nivel global. En la siguiente sección, se exponen aspectos característicos de la formación a nivel posgradual para el caso de la disciplina, en específico para Colombia. Por último, se recogen las percepciones de empresarios y empleados del sector asegurador, así como de estudiantes y egresados del programa de especialización en Riesgos y seguros del Politécnico Grancolombiano, para determinar la pertinencia del contenido curricular del programa y servir de insumo para desarrollar las actualizaciones curriculares pertinentes.

\section{DISEÑO METODOLÓGICO}

Los resultados presentados en este documento resultan del desarrollo de una investigación de tipo descriptivo, toda vez que buscan identificar las necesidades en términos de la formación posgradual en riesgos, en el contexto de las organizaciones, y contrastarlas con la oferta académica existente.

Para estructurar el conjunto de riesgos que preocupan a las organizaciones, se cruzaron los elementos comunes determinados por 
diferentes organismos y publicaciones especializadas en la gestión de este fenómeno en las empresas. Es así como se presentan las clasificaciones hechas por la Fundación Mapfre, el Foro Económico Mundial y el Barómetro de riesgos de Allianz Global Corporate.

Para poder determinar la presencia o no de un mismatch entre las preocupaciones de las organizaciones en términos de riesgos y la oferta académica posgradual en el país, se hizo un análisis de los planes de estudio de la oferta de programas que tratan este tema de forma integral, contrastándolos con el inventario previamente establecido.

Por último, y dado que una de las motivaciones de esta investigación fue la actualización de los contenidos curriculares del programa de especialización en Gerencia de Riesgos y seguros del Politécnico Grancolombiano, se validó mediante un instrumento de opinión la percepción de los grupos de interés del programa (empresarios y empleados del sector asegurador, estudiantes y egresados, y también egresados de otros programas de posgrado en riesgos), acerca de la relevancia que tiene para sus organizaciones y desempeño laboral el aprendizaje de los riesgos identificados en la etapa anterior. La encuesta se desarrolló vía web, mediante un formulario de Microsoft, que estuvo abierta durante 20 días, obteniendo un total de 200 observaciones. Se debe resaltar que los riesgos identificados, luego de realizada la encuesta, fueron por último validados mediante entrevista realizada a un presidente de compañía aseguradora, dos directores de riesgo de compañías de seguros y dos gerentes/dueños de empresas, encargados de la gestión de riesgos.

De acuerdo con la metodología planteada, este documento hace uso de fuentes primarias y secundarias, estas últimas provenientes de diferentes bases de datos como Web of Science, Scopus, Jstor, Scielo, entre otras publicaciones especializadas. Las palabras clave utilizadas en la búsqueda, en inglés y en español, fueron las siguientes: gestión de riesgos, administración de riesgos, educación, riesgos asegurables, clasificación de riesgos, ya sea que se encontraran en el título, en el resumen y/o en el cuerpo del texto, basándose la selección de los artículos en la relevancia del título y/o resumen.

\section{DISCUSIÓN Y RESULTADOS}

\section{¿Cuáles riesgos importan a las organizaciones?}

El estudio del riesgo según Gómez (2014), desde las diferentes disciplinas, implica la preocupación por la manifestación de ciertos eventos y la anticipación a su ocurrencia. El análisis de riesgos implica individualizar el evento, identificar sus causas para anticiparse a su ocurrencia o prepararse ante los efectos una vez ha acontecido. Esta perspectiva del análisis de dichos fenómenos es resultado de la evolución de la visión del hombre (y de las organizaciones), en su papel dentro de este campo de estudio, partiendo de una posición pasiva, meramente limitada al análisis de la situación una vez ocurría y a la acción posterior al evento, hasta el 
reconocimiento del papel protagonista del sujeto como contribuyente, por acción u omisión al suceso.

La gestión de riesgos siempre ha hecho parte de manera más o menos formal del manejo de las organizaciones (etimológicamente hablando, la palabra riesgo se asocia con risco, peñasco escarpado que para los marineros medievales representaba un peligro en alta mar (Gómez, 2014)), y en la historia reciente, como parte de la política pública de los gobiernos.

Para el caso colombiano, no es sino hasta la destrucción del municipio de Armero, como consecuencia de la avalancha generada por el volcán Nevado del Ruiz en el año 1985, que se hace manifiesta la necesidad de contar con un sistema que permitiera coordinar la respuesta a este tipo de eventualidades, pero solo en el año 1992, con el decreto 1547, se establece el Fondo Nacional de Calamidades. Con la Ley 46 de 1988 se da origen al Sistema Nacional para la Prevención y Atención de Desastres, como órgano adscrito a la Presidencia de la República para tal fin, que evoluciona hasta el punto de que actualmente, la gestión del riesgo hace parte de la política pública, tal como está consagrado en la Ley 1523 de 2012 y que define como responsables de la gestión de riesgo a todas las autoridades y habitantes del territorio nacional.

En el ámbito de las organizaciones, en el año 1990, la Fundación Mapfre[1] realiza una clasificación formal de riesgos en la empresa, en la que presenta un listado de riesgos que aún hoy permanece vigente, y que es de obligatoria referencia para quienes se encarguen de su gestión (ver tabla 1) (Aventín, 1990). 


\begin{tabular}{|c|c|}
\hline Riesgo & Relacionado con \\
\hline De producción & $\begin{array}{l}\text { Equipos usados para la fabricación, su obsolescencia, el almacenamiento de } \\
\text { materias primas y productos terminados, proveedores, capacidad de } \\
\text { producción. }\end{array}$ \\
\hline De ventas & $\begin{array}{l}\text { Productos y servicios ofrecidos, publicidad, errores de producto, transporte, } \\
\text { importación, exportación, competencia, cadena de suministro. }\end{array}$ \\
\hline Financieros & Insolvencia, inversiones, endeudamiento, crédito. \\
\hline Del medio ambiente & $\begin{array}{l}\text { Posición geoestacionaria de la planta, relaciones laborales y clima laboral, } \\
\text { estabilidad política, condiciones micro y macroeconómicas. }\end{array}$ \\
\hline De dirección & $\begin{array}{l}\text { Establecimiento de costos, precios, utilidades, medios de distribución, } \\
\text { reputación, crecimiento, expansión, solvencia financiera y estabilidad } \\
\text { económica, gestión de procesos. }\end{array}$ \\
\hline $\begin{array}{l}\text { Personales o } \\
\text { laborales }\end{array}$ & Accidente, el secuestro, la muerte, enfermedad. \\
\hline $\begin{array}{l}\text { Asociados a daños } \\
\text { materiales }\end{array}$ & $\begin{array}{l}\text { Fenómenos de la naturaleza (lluvias torrenciales, los terremotos o erupciones } \\
\text { volcánicas), la caída de objetos, conflictos bélicos, acciones políticas, guerras, } \\
\text { revueltas, rebelión, sabotaje, atentados. }\end{array}$ \\
\hline Tecnológicos & $\begin{array}{l}\text { El fuego o la explosión, el corto circuito o la sobretensión, desgaste, corrosión, } \\
\text { falta de mantenimiento o deficiente, defectos en la construcción, contaminación } \\
\text { ambiental, infidelidad del personal. }\end{array}$ \\
\hline Judiciales & $\begin{array}{l}\text { Asociados a contratos, cargas sociales, negligencia, propiedades físicas, } \\
\text { custodia de bienes de terceros, productos defectuosos, transporte de } \\
\text { mercancías peligrosas, accidentes derivados de la conducción de vehículos, la } \\
\text { contaminación ambiental por los procesos de producción o el destino de } \\
\text { residuos. }\end{array}$ \\
\hline
\end{tabular}

\section{Tabla 1.}

Clasificación de riesgos en la empresa Fuente: Fundación Mapfre (1990).

Sin embargo, los estándares para la gestión de riesgo han sido diseñados también por diferentes instituciones internacionales, como el Project Management Institute, el National Institute of Standards and Technology de los Estados Unidos, sociedades actuariales y la International Organization for Standardization, ISO. Esta última como referente en la gestión de calidad. Es así como para el año 2015 se presenta la nueva versión del Sistema de Gestión de la Calidad ISO 9001, donde se puede evidenciar la importancia que los riesgos han tomado para los entes certificadores, al involucrar un capítulo completo sobre estos bajo un modelo denominado Pensamiento Basado en Riesgos. La norma invita a identificar los riesgos, analizarlos, priorizarlos y planificar acciones para tratarlos, con el fin de que las organizaciones puedan alcanzar sus objetivos, promocionando el uso de acciones preventivas ante los riesgos, al tiempo que se garanticen acciones de mejora continua para todos los procesos.

Martínez y Blanco (2017), resaltan la relevancia que tiene la gestión integral de los riesgos en la actualidad, teniendo en cuenta las crisis financieras globales, la necesidad de la evaluación de los procesos y de contar con empresas más transparentes ante los ojos del público en general y sus accionistas. Proponen abordar riesgos tales como el fraude, la quiebra de clientes, cambios regulatorios, riesgos operativos, de la industria, institucionales y del país, los reputacionales, la responsabilidad social corporativa y conjugar un inventario de riesgos que disminuyan la capacidad para alcanzar los objetivos organizacionales. Sin embargo, 
los riesgos propuestos por estas autoras corresponderían a riesgos especulativos, sin tener en cuenta otros riesgos que podrían estar dentro del inventario de riesgos empresariales y que podrían ser asegurables, como pueden ser el riesgo de enfermedad, los de la naturaleza y de responsabilidad civil por daños a terceros.

En este sentido, en el décimo cuarto reporte del Foro Económico Mundial, entregado en enero de 2019 por las compañías Marsh y McLennan, en asocio con la aseguradora Zurich Group, se plantean una serie de riesgos que aquejan a las empresas a nivel mundial (ver figura 1).

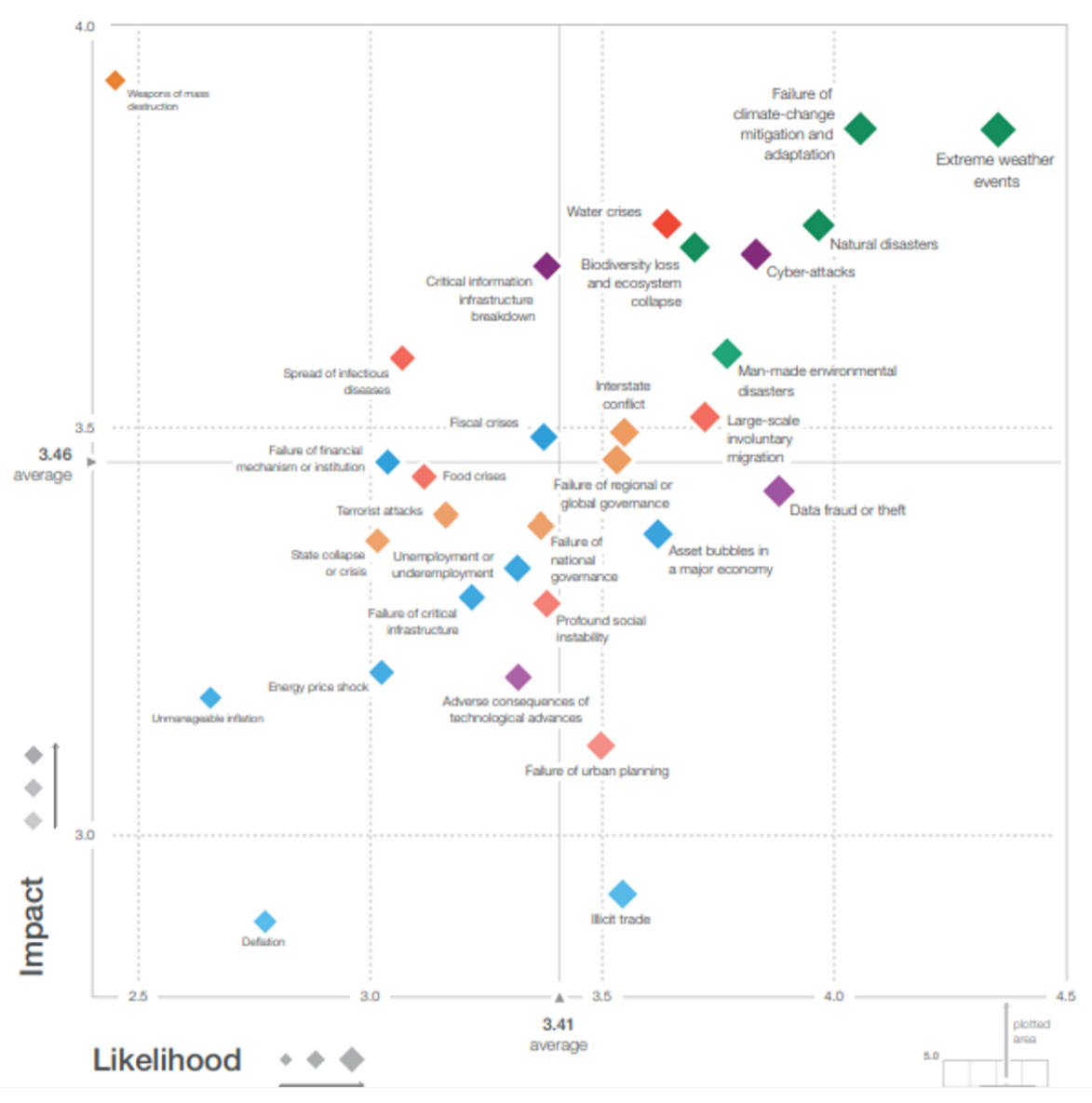

Figura 1.

Panorama de Riesgos Globales

Fuente: World Economic Forum (2019).

Este informe expresa que los riesgos que mayor probabilidad de ocurrencia e impacto tienen sobre una organización son los eventos climáticos extremos, los desastres naturales, la crisis por falta de agua, la pérdida de la biodiversidad, el colapso de los ecosistemas, los ataques cibernéticos, la pérdida de información o el mal uso de ella, así como daños hechos por el hombre a su entorno, entre otros riesgos. Algunos de estos, como el efecto de invernadero, no son asegurables, pero sí algunas de sus consecuencias. Por ejemplo, las afectaciones climáticas (lluvia, sequía, granizo, inundaciones, heladas) sobre los procesos y productos agrícolas, o sobre los bienes de una empresa, se cuentan cubiertos por pólizas de seguros. 
Los desastres naturales, en cambio, sí se encuentran cubiertos por pólizas de seguros, y pueden ocasionar pérdidas humanas y materiales: riesgos tales como la erupción volcánica, el terremoto, inundaciones, tsunamis, deslizamientos de tierra, los huracanes, entre otros, son reconocidos como riesgos de la naturaleza.

Por su parte, la encuesta sobre Percepción del Riesgo Cibernético en Latinoamérica 2019, de Marsh en asocio con Microsoft, demuestra que este riesgo viene siendo muy importante para las empresas, cerca del $73 \%$ de las organizaciones encuestadas lo clasifica como una de sus cinco principales preocupaciones. El informe, además, resalta:

El riesgo cibernético ha pasado del robo de datos y la preocupación por la privacidad a esquemas más sofisticados que pueden interrumpir la operación en empresas, industrias, cadenas de suministro y naciones, costándole a la economía miles de millones de dólares en cada sector. La dura realidad a la que las organizaciones deben enfrentarse es que el riesgo cibernético no puede eliminarse, por lo tanto, debe gestionarse a través de la identificación, mitigación y transferencia. (Marsh, 2019, p. 1)

Algunas de las consecuencias de este riesgo vienen siendo cubiertas por pólizas de seguros, permitiendo de algún modo recuperar las pérdidas que las empresas pueden sufrir por el robo y manipulación inadecuada de bases de datos de sus clientes, la destrucción de los datos, la falla de sistemas importantes para las organizaciones, la posible interrupción del negocio o el fraude por computador.

Otro informe que permite hacer un panorama acerca de los riesgos que preocupan a las organizaciones es el "Barómetro de Riesgos", publicado por Allianz Global Corporate \& Specialty (2019). Los informes compartidos de 2014 a 2019 permiten observar que se han producido cambios significativos en el ambiente empresarial, pero la mayoría de los riesgos que preocupan en mayor medida a los empresarios se han mantenido o fusionado. En la figura 2 se encuentra un resumen del comportamiento de los riesgos, según los expertos consultados por Allianz. 


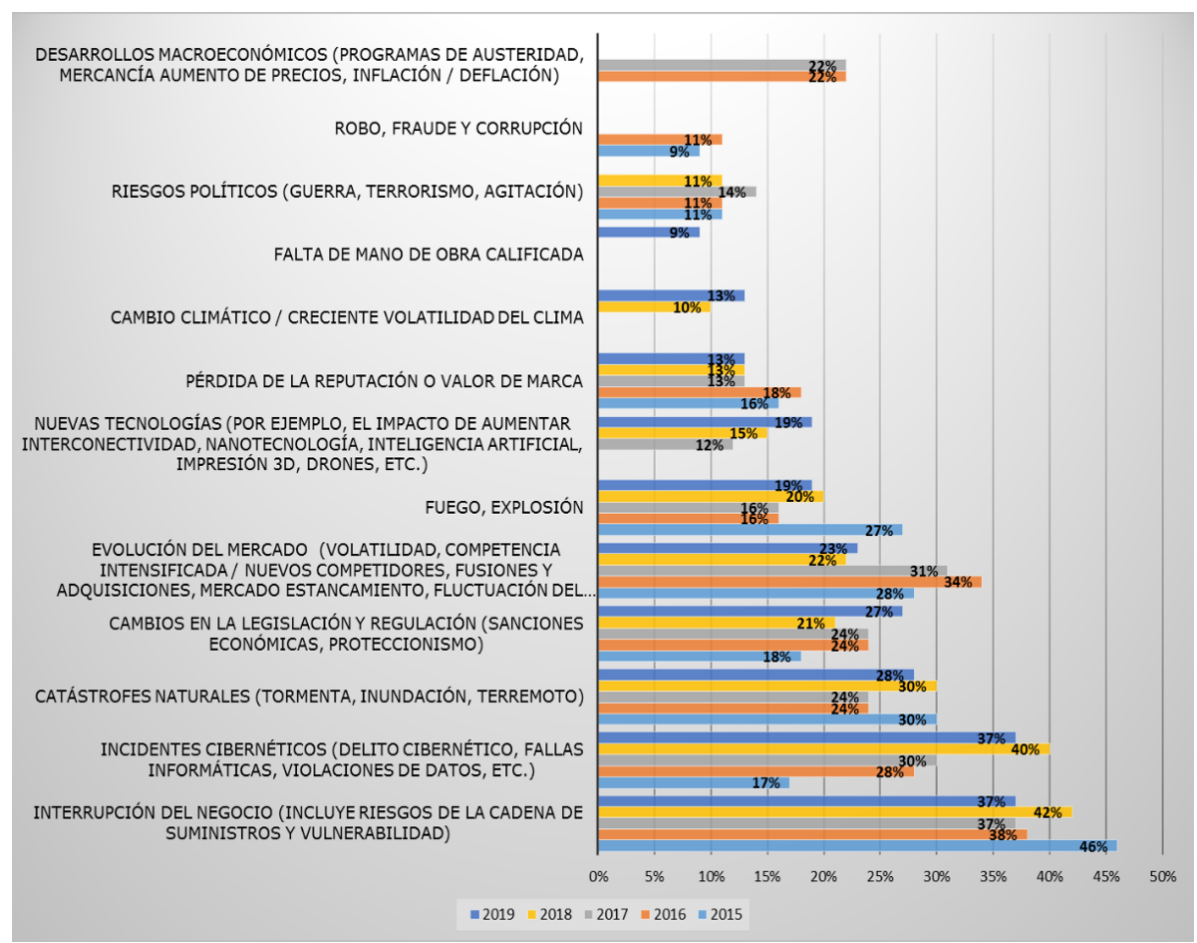

Figura 2.

Variación de los riesgos, de 2015 a 2019, según el Barómetro de Riesgos de Allianz Global Corporate \& Specialty

Fuente: elaboración propia con información de Allianz Global Corporate \& Specialty.

Para el año 2019, los primeros cinco riesgos fueron la interrupción del negocio (que incluye riesgos de la cadena de suministros y vulnerabilidad), los incidentes cibernéticos (delito cibernético, fallas informáticas, violaciones de datos, etc.), las catástrofes naturales (tormenta, inundación, terremoto), cambios en la legislación y regulación (sanciones económicas, proteccionismo), y evolución del mercado (volatilidad, competencia intensificada / nuevos entrantes, fusiones y adquisiciones, mercado estancamiento, fluctuación del mercado).

La interrupción del negocio puede derivarse de daños materiales causados por riesgos de la naturaleza o por la intervención del hombre, como el sabotaje, los daños mal intencionados de terceros, las huelgas, motines, la guerra, conmoción civil o popular, entre otros riesgos. En sí, cuando una interrupción se deriva de daños materiales se pueden causar pérdidas económicas que afectan la utilidad de una empresa; estas pérdidas se encuentran cubiertas por pólizas de seguros, de otro modo se requiere de planes de contingencia o de continuidad que eviten la paralización de las operaciones.

Para el caso de enfermedad, como puede ser la acción del Covid-19 en el año 2020, no existen coberturas de pólizas de seguros que permitieran mitigar las pérdidas ocasionadas por la interrupción del negocio a consecuencia de esta pandemia, por lo que muchas empresas dejaron de funcionar o se basaron en sus planes de continuidad de negocio para mitigar sus pérdidas, basados en la experiencia de los expertos en administración de riesgos. 
Con respecto a incidentes cibernéticos, se han mencionado situaciones cubiertas por pólizas de seguros, aunque temas como el acoso, la pornografía infantil, los agravios y calumnias, la usurpación de personas, no lo están, al igual que actividades como el hacking ${ }^{[2]}$, phishing ${ }^{[3]}$ o malware ${ }^{[4]}$, aunque sí la responsabilidad civil derivada del mal uso de la información obtenida mediante estos métodos, sin que esto libere extrapatrimonialmente al afectado por el mal uso de su información.

Los cambios en la legislación, así como la evolución del mercado, no son asegurables, ambos se consideran riesgos especulativos. Adicional a esto, existe un par de nuevos actores que poco a poco están generando cambios en el mercado laboral: los robots y la inteligencia artificial, lo que se ha denominado la Cuarta Revolución Industrial. Todas las que han sido denominadas revoluciones industriales han traído consigo el mismo riesgo para los seres humanos: desempleo, y este con un ingrediente adicional, su acelerada propagación: "Los mismos avances que ofrecen la visión de un futuro extraordinario, plantean amenazas inimaginables: los riegos existenciales modernos." (Díaz, 2017, s.p.)

\section{La formación en riesgos a nivel posgradual}

Como se ha señalado en párrafos anteriores, la gestión del riego en las organizaciones implica una comprensión amplia de factores que pueden afectar la continuidad del negocio. Una de las principales preocupaciones de las organizaciones es la gestión integral de los riesgos, toda vez que dicha gestión tiene una relación estrecha con el mantenimiento y el aumento del valor de los recursos humanos, físicos y financieros de una empresa, por lo que ninguna decisión carece de un aspecto de gestión de riesgos (Corbett, 2004).

Sin embargo, una de las principales barreras que se pueden identificar en la enseñanza de gestión de riesgos a nivel posgradual tiene que ver con la comprensión del carácter de integralidad del concepto riesgo. Como señalan Acharyya y Brady (2014), cada rama de las ciencias sociales tiene una unidad de observación diferente, desde la economía que analiza los comportamientos riesgosos a nivel individual; la estrategia que mira el riesgo desde actividades grupales, la sociología que lo analiza como un fenómeno social y, dentro de cada una de estas grandes áreas se pueden exponer innumerables objetos de análisis.

A una conclusión similar llega Chávez (2018), quien indica que dependiendo del enfoque que se brinde a su definición, y al contexto en el que se emplee, puede tener diferentes interpretaciones y posibles usos inadecuados del término, generando discusión y desconcierto en las aulas de clase desde el punto de vista teórico.

Stulz (1996), indica incluso cómo a nivel de finanzas corporativas puede o debería utilizarse la gestión del riesgo, específicamente el riesgo financiero, como un elemento de generación de valor agregado para las compañías, cuando las empresas pueden tomar posiciones de especulación 
con base en información especializada que se deriva del conocimiento profundo que se tiene sobre la actividad económica.

Por otro lado, la enseñanza de la gestión del riesgo y de los seguros involucra una serie de desafíos asociados, primero con la rapidez con la que este tema cambia, evoluciona y se sofistica, y segundo, con la continua aparición de nuevas fuentes de incertidumbre (Hoyt, Dumm, \& McCullough, 2010).

Para el caso colombiano, y como se muestra en el gráfico comparativo de la figura 3, se puede observar que mientras se desarrollan diferentes estudios y enfoques entorno al análisis de los riesgos, la oferta de formación en este tema ha permanecido más o menos estática, lo que puede generar disparidades entre las necesidades de las organizaciones, en términos de contar con una mano de obra altamente capacitada y la oferta de formación.

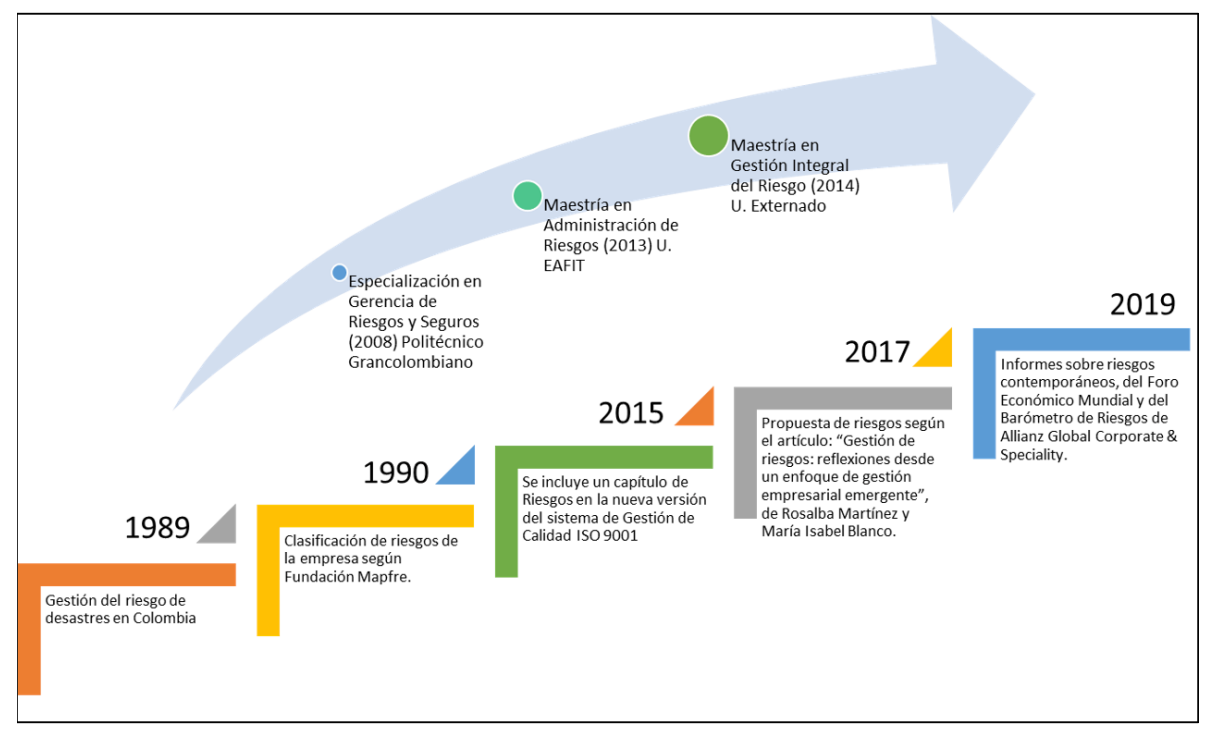

Figura 3.

Comparación cronológica entre estudios sobre riesgos y las propuestas educativas.

Fuente: elaboración propia.

Los riesgos que importan a las organizaciones y los planes de estudio: comparación

La comparación entre los riesgos a nivel mundial y los riesgos abordados en programas de posgrado ya existentes en Colombia permitirá determinar aquellos que ya están siendo cubiertos por la oferta académica actual e identificar los que puedan requerir de estudio mediante nuevos programas de posgrado.

El Foro Económico Mundial y Allianz mantienen el enfoque de riesgos del presente documento: desde el punto de vista económico; el primero destaca los principales riesgos que pueden afectar al mundo en los siguientes diez años de la publicación de sus resultados, abordando especialmente problemas geopolíticos, económicos y acuerdos comerciales. Allianz, por su parte, aborda los riesgos por sectores, teniendo en cuenta 22 sectores económicos, ver tabla 2. 


\begin{tabular}{|c|c|c|c|c|c|}
\hline $\begin{array}{c}\text { Fundación } \\
\text { Mapfre (1990) }\end{array}$ & $\begin{array}{c}\text { Martínez \& } \\
\text { Blanco (2017) }\end{array}$ & $\begin{array}{c}\text { Foro } \\
\text { Económico } \\
\text { Mundial } \\
\text { (2919) }\end{array}$ & $\begin{array}{c}\text { Barómetro de } \\
\text { riesgos (2919) } \\
\text { Allianz Global } \\
\text { Corporate }\end{array}$ & $\begin{array}{c}\text { Externado } \\
(2019) \\
\text { EAFIT (2019) }\end{array}$ & $\begin{array}{c}\text { Politécnico } \\
\text { Grancolombiano } \\
\text { (2019) }\end{array}$ \\
\hline De producción & & & & $\begin{array}{c}\text { Transferencia de } \\
\text { riesgos vía } \\
\text { seguros; } \\
\text { Proyectos }\end{array}$ & \multirow{3}{*}{ Reales } \\
\hline $\begin{array}{l}\text { Asociados a } \\
\text { daños } \\
\text { materiales }\end{array}$ & & $\begin{array}{l}\text { Eventos } \\
\text { climáticos } \\
\text { extremos, } \\
\text { desastres } \\
\text { naturales }\end{array}$ & $\begin{array}{l}\text { Catástrofes } \\
\text { naturales }\end{array}$ & $\begin{array}{l}\text { Desastres } \\
\text { naturales }\end{array}$ & \\
\hline Tecnológicos & $\begin{array}{c}\text { Riesgos } \\
\text { operativos }\end{array}$ & & $\begin{array}{l}\text { Fuego, } \\
\text { explosión }\end{array}$ & Informáticos & \\
\hline $\begin{array}{c}\text { Personales o } \\
\text { laborales }\end{array}$ & & & & Laborales & Personas \\
\hline \multirow[t]{2}{*}{ Judiciales } & Fraude & & $\begin{array}{l}\text { Interrupción del } \\
\text { negocio }\end{array}$ & $\begin{array}{l}\text { Continuidad de } \\
\text { negocio }\end{array}$ & \multirow[b]{2}{*}{ Patrimoniales } \\
\hline & $\begin{array}{c}\text { La } \\
\text { responsabilidad } \\
\text { social } \\
\text { corporativa }\end{array}$ & $\begin{array}{l}\text { Ataques } \\
\text { cibernéticos }\end{array}$ & $\begin{array}{l}\text { Incidentes } \\
\text { cibernéticos }\end{array}$ & & \\
\hline De dirección & Reputacionales & $\begin{array}{l}\text { Pérdida o mal } \\
\text { uso de la } \\
\text { información }\end{array}$ & $\begin{array}{l}\text { Pérdida de la } \\
\text { reputación o } \\
\text { valor de la } \\
\text { marca }\end{array}$ & $\begin{array}{l}\text { Alta dirección, } \\
\text { reputación, legal } \\
\text { y contractual, } \\
\text { seguridad }\end{array}$ & \\
\hline Financieros & & & & Finanzas & Financieros \\
\hline De ventas & $\begin{array}{l}\text { Quiebra de } \\
\text { clientes }\end{array}$ & & $\begin{array}{l}\text { Evolución del } \\
\text { mercado }\end{array}$ & & \\
\hline \multirow[t]{4}{*}{$\begin{array}{l}\text { Del medio } \\
\text { ambiente }\end{array}$} & $\begin{array}{l}\text { De la industria, } \\
\text { institucionales y } \\
\text { del país }\end{array}$ & $\begin{array}{c}\text { Crisis por falta } \\
\text { de agua, pérdida } \\
\text { de biodiversidad, } \\
\text { colapso de } \\
\text { ecosistemas }\end{array}$ & $\begin{array}{c}\text { Cambio } \\
\text { Climático }\end{array}$ & & \\
\hline & $\begin{array}{l}\text { Cambios } \\
\text { regulatorios }\end{array}$ & & $\begin{array}{l}\text { Cambios en la } \\
\text { legislación y } \\
\text { regulación. }\end{array}$ & & \\
\hline & & & $\begin{array}{l}\text { Nuevas } \\
\text { tecnologías }\end{array}$ & & \\
\hline & & $\begin{array}{l}\text { Daños del ser } \\
\text { humano a su } \\
\text { entorno }\end{array}$ & & & \\
\hline
\end{tabular}

Tabla 2.

Tabla comparativa de riesgos identificados usados de consulta

Fuente: elaboración propia.

La tabla 2 presenta una comparación entre los riesgos identificados y las mallas curriculares de programas de posgrado en Colombia, de ciudades principales como Bogotá y Medellín (porque allí se encuentran las universidades Externado de Colombia y EAFIT), cuyo eje central es la enseñanza en gestión y/o administración de riesgos, y que se asemejan al programa de especialización en Gerencia de Riesgos y seguros del Politécnico Grancolombiano[5].

La tabla 2 permite establecer que en la actualidad los estudios de maestría en riesgos están relacionados con la gestión del riesgo, su administración, los riesgos laborales, de seguridad, transferencia de riesgos a pólizas de seguros, riesgos legales y contractuales, operativos y de continuidad de negocio, riesgo financiero, informáticos y los asociados a los proyectos y a desastres naturales, de acuerdo con las propuestas de la Universidad EAFIT y la Universidad Externado de Colombia.

Los programas académicos asociados a riesgos requieren de espacios que permitan el descubrimiento y aprendizaje sobre nuevos riesgos, para citar un ejemplo, lo ocurrido con la enfermedad del Covid19 en 2020; tal vez las universidades debieron ser las fuentes de información primaria para el mundo empresarial colombiano, que detectara con sus investigaciones este tipo de riesgos eventuales, que aunque no son nuevos, 
se pierden muchas veces de los radares de riesgos de entes nacionales e internacionales, o son relegados por otros riesgos que se consideran de mayor relevancia, muchas veces por su frecuencia y su impacto a corto plazo.

De ningún modo se pretende en este documento demeritar el esfuerzo que hacen las universidades para que sus estudiantes aprendan sobre riesgos y su gestión, lo que se pretende es que se recuerde que el entorno de los riesgos es dinámico, y que los programas académicos debieran poder adaptarse, en sus contenidos, a esas dinámicas.

\section{Formación en riesgos y percepción de los grupos de interés}

De las 200 personas encuestadas, 189 expresaron vivir en Colombia, 102 pertenecen al sector asegurador y de ellos uno (1) era vicepresidente de compañía, 27 eran directores, 14 gerentes y cinco (5) coordinadores. De los 98 encuestados que no pertenecen al sector asegurador, el $44 \%$ ejercían cargos de dirección: tres (3) eran presidentes de empresa, uno (1) vicepresidente, 11 directores, 21 eran gerentes y siete (7) coordinadores.

El $77 \%$ de los encuestados expresaron que aprender sobre riesgos era importante por razones específicas, en las que se resalta el conocer acerca de este tema para "idear controles y mitigar las pérdidas", temas como "prepararse para el futuro", "la prevención" y "situaciones de riesgo frecuentes, en las que vivimos", hacen parte de las razones más relevantes que el grupo considera importante para aprender sobre riesgos. El 22\% de los encuestados respondieron solo que sí era importante aprender sobre riesgos, sin ninguna razón especial (ver figura 4), y de las 200 respuestas una (1) persona afirmó no ser importante aprender al respecto. 


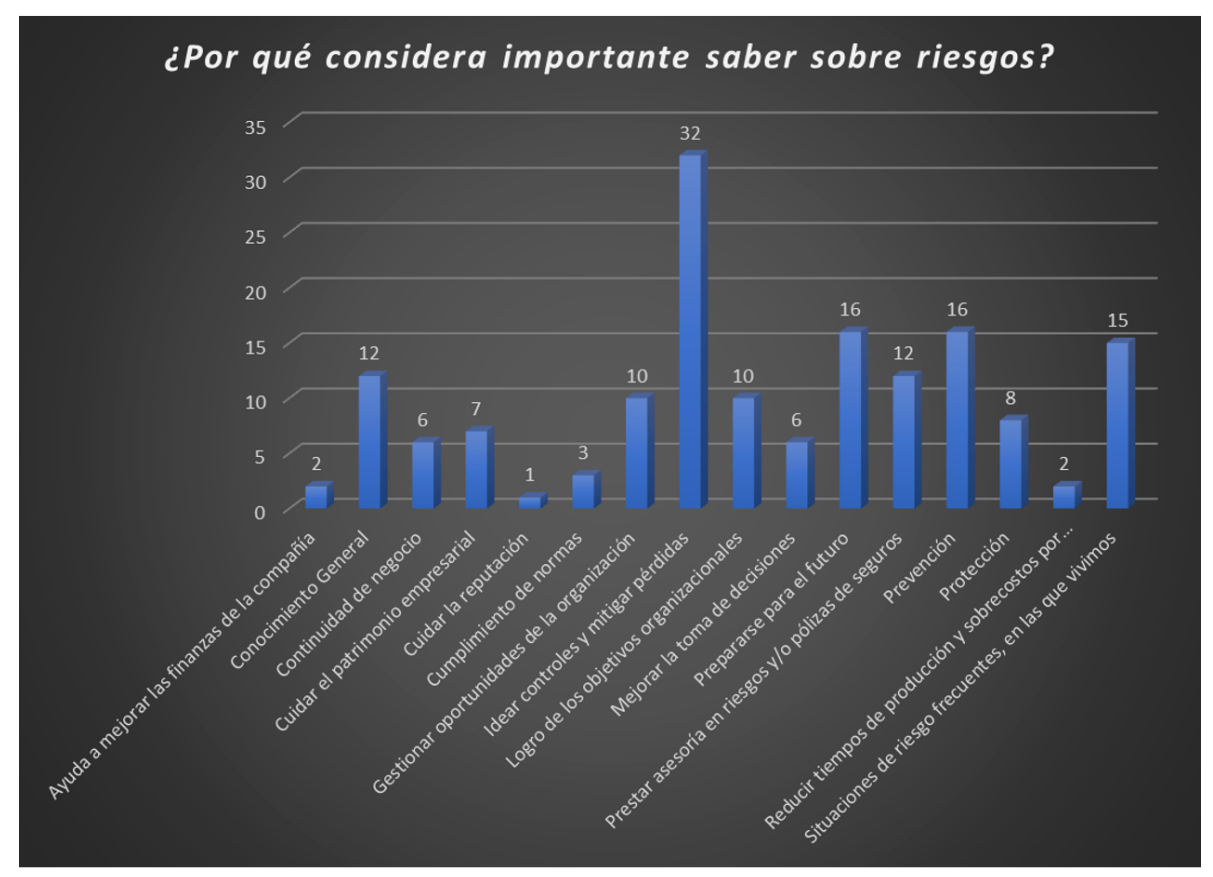

Figura 4.

¿Considera importante aprender sobre riesgos? ¿Por qué?

Fuente: elaboración propia.

Cuando se analiza por perfil del encuestado, se tiene que los cinco temas sobre los que les gustaría aprender a los directivos de las organizaciones fueron: riesgos asociados a la dirección (42 respuestas), los riesgos financieros (40 respuestas), el riesgo cibernético (39 respuestas), riesgos asociados a las ventas (30 respuestas) y los asociados a la producción (27 respuestas). Los relacionados con pólizas de seguros (25 respuestas), se encuentran en el puesto 6 de 12.

Para las personas que no ocupan cargos de dirección dentro de sus organizaciones, los cinco temas de mayor interés fueron: riesgo cibernético ( 53 respuestas), financiero ( 52 respuestas), riesgos asociados a pólizas de seguros ( 49 respuestas), riesgos de dirección (42 respuestas) y tecnológico (34 respuestas). Más atrás se encuentran, en este orden, el riesgo asociado al proceso de ventas, riesgo de medio ambiente social y los riesgos judiciales.

En cuanto a la normatividad relacionada con la gestión de riesgos, 123 encuestados respondieron la pregunta, 14 de ellos afirmaron no conocer normas relacionadas, 69 dieron ideas de lo que les gustaría aprender con respecto a riesgos, sin nombrar alguna norma en particular, y 40 de los encuestados mencionaron lo que consideraban modelos o normas de administración de riesgos sobre las que les gustaría aprender:

1. COSO - Committee of Sponsoring Organizations of the Treadway.

2. LEY 1523 DE 2012, sobre gestión del riesgo de desastres y el sistema nacional colombiano de gestión del riesgo de desastres.

3. ISO 27000 - Gestión y seguridad de la Información.

4. ISO 28001 - Sistema de gestión de seguridad de la cadena de suministro.

5. ISO 31000 - Directrices para la gestión de riesgos. 
6. QRA - Cuantificar la Evaluación del Riesgo.

7. SARC - Sistema de Administración del Riesgo de Crédito.

8. SARL - Sistema de Administración de Riesgos de Liquidez.

9. SARLAFT - Sistema de Administración de Riesgo de Lavado de Activo y Financiación del Terrorismo.

10. SARM - Sistemas de Administración de Riesgos de Mercado.

11. SARO - Sistema de Administración de Riesgo Operativo.

12. Marco de Sendai para la reducción del riesgo de desastres

13. SGSI - Sistema de Gestión de Seguridad de la Información.

14. SIAR - Sistema Integral de Administración de Riesgos.

\section{CONCLUSIONES Y DISCUSIÓN}

El carácter dinámico de los riesgos, es decir, los existentes y la aparición de nuevos, que a su vez evolucionan como resultado de fenómenos asociados a la modernidad (cambios tecnológicos, el internet de las cosas, los riesgos cibernéticos, la inteligencia artificial, la robótica, cambio climático, etc.), exige desde las comunidades educativas contar con programas que permitan formar profesionales que sean capaces de administrar las organizaciones en estos nuevos contextos, teniendo en cuenta todo lo que incluye la gestión de riesgos.

Observando los resultados del instrumento, los riesgos evaluados por entidades como el Foro Económico Mundial, Allianz, Fundación Mapfre, instituciones educativas como el Politécnico Grancolombiano, el Externado y EAFIT, y autores como Martínez y Blanco (2017), se puede afirmar que los riesgos más relevantes que debieran tenerse en cuenta en un programa de posgrado sobre riesgos en las organizaciones son:

1. Riesgo cibernético.

2. Interrupción del negocio.

3. Riesgos asociados a la dirección de empresas.

4. Riesgo financiero.

5. Riesgos asegurables asociados a producción, tecnológicos, personas, legales, cambios regulatorios, judiciales.

6. Riesgo operacional asociado a catástrofes naturales, eventos climáticos extremos.

7. Riesgos asociados a la evolución del mercado, de la industria.

8. Riesgo antrópico.

Estos riesgos deben ponerse a consideración de las instituciones universitarias, pudiendo tener en cuenta las consideraciones hechas por cinco expertos en riesgos para el desarrollo de los contenidos:

a) Los riesgos derivados de malestares sociales, observando el número de manifestaciones públicas que ocurrieron en Colombia, China, Chile, entre otros, en el año 2019.

b) Abordar el riesgo reputacional,

c) Siendo que, por definición, los riesgos pueden ser de carácter natural y artificial, y que este último está relacionado directamente con el accionar del ser humano sobre el planeta, los entrevistados expresan la necesidad de abordar el riesgo antrópico, así como incluir la administración de 
riesgos en proyectos, para aprender a maximizar oportunidades y tratar de manera adecuada eventos negativos asociados a nuevos desarrollos e innovación;

d) Fusionar los riesgos financieros con los riesgos asociados a la dirección de empresas, teniendo en cuenta que muchas de las decisiones que toma la alta gerencia, además de asumir la visión y la misión empresarial, y sus objetivos estratégicos, evalúan también las necesidades financieras y económicas de la organización, con el fin de alcanzar esos objetivos.

Con base en los resultados obtenidos, se hace manifiesta la necesidad de reestructurar contenidos curriculares que se desarrollan en programas posgraduales asociados a la gestión del riesgo, basados en las relaciones y necesidades de las organizaciones; en específico, el programa de especialización en Gerencia de Riesgos y seguros de la Institución Universitaria Politécnico Grancolombiano, que cubre solo dos de los ocho riesgos que más interesan a las personas, tanto a nivel nacional como a nivel mundial.

Diseñar un programa de posgrado que permita abordar todos los riesgos mencionados, o realizar ajustes curriculares a los ya existentes, es un paso inevitable hacia la coincidencia de la oferta académica con las necesidades de las organizaciones.

\section{REFERENCIAS}

Acharyya, M., \& Chris, B. (2014). Designing an enterprise risk management curriculum for business studies: insights from a pilot program. Risk Management and Insurance Review, 17(1), 113-116. doi:10.1111/ rmir.12019

Allianz Global Corporate \& Specialty. (2019, enero 18). Allianz Global Corporate \& Specialty. Recuperado de agcs.allianz.com: https://www.agcs .allianz.com/assets/PDFs/Reports/Allianz_Risk_Barometer_2019.pdf

Aventín. (1990). Clasificación de riesgos en la empresa. Gerencia de riesgos, 45-54. Recuperado de: https://www.fundacionmapfre.org/documentaci on/publico/es/catalogo_imagenes/grupo.cmd?path $=1029076$

Chávez, S. (2018). El concepto de riesgo. Recursos Naturales y Sociedad, 32-52.

CiberDerecho. (2019, febrero 28). Ciber Derecho. Recuperado de ciberderecho.com: http://www.ciberderecho.com/que-es-el-hacking/

Congreso de Colombia. (2012, Abril 24). Ley 1523 de 2012. Por la cual se adopta la politica nacional de gestión del riesgo de desastres y se establece el Sistema Nacional de Gestión del Riesgo de Desastres y se dictan otras disposiciones. Bogotá. Recuperado de: https://www.funcionpublica.gov.co/eva/gestorn ormativo/norma.php?i=47141

Corbett, R. (2004). A View of the Future of Risk Management. Risk Management: An International Journal, 51-56.

Diaz, B. (2017, marzo 30). BBVA. Recuperado de bbva.com: https://www.bbv a.com/es/riesgos-existenciales-futuro-la-humanidad/

Gómez, A. (2014). Marco conceptual y legal sobre la gestión de riesgo en Colombia: aportes para su implementación. (S. N. Salud, Ed.) Monitor 
Estratégico, 4-11. Recuperado de: https://www.minsalud.gov.co/sites/rid /Lists/BibliotecaDigital/RIDE/IA/SSA/Articulo\%201.pdf

Grupo Lucía Botín. (2018). Secretaría de Educación Pública. Recuperado de: luciabotin.mx: https://luciabotin.mx/pages/blog/entradas/delitoscibern eticos.php

Head, G. (1973). Teaching Risk Management as a Decision Process. A Review of the New I.I.A. Program in Risk Management. The Journal of Risk and Insurance, 40(1), 149-151. doi:10.2307/252019

Hoyt, R., Dumm, R., \& McCullough, K. (2010). Risk Management Case Project. Risk Management and Insurance Review, 147-159.

Marsh. (2019). Encuesta de Percepción del Riesgo Cibernético en Latinoamérica. México. Recuperado de: https://www.anuarioseguros.lat/admin/storage /files/MARSH_2.pdf

Martínez, R., \& Blanco, M. (2017). Gestión de riesgos: reflexiones desde un enfoque de gestión empresarial emergente. Revista Venezolana de Gerencia, 693-711.

Rivero, M. (2019). Info Spyware. Recuperado de infospyware.com: https://ww w.infospyware.com/articulos/que-es-el-phishing/

Rivero, M. (2019). Info Spyware. Recuperado de infospyware.com: https://ww w.infospyware.com/articulos/que-son-los-malwares/

Stulz, R. (1996). Rethinking Risk Management. Journal of Applied Corporate Finance, 9(3), 8-24.

World Economic Forum. (2019). The Global Risks Report 2019. Geneva: World Economic Forum. Recuperado de: http://www3.weforum.org/docs/WE F_Global_Risks_Report_2019.pdf 